PROCEEDINGS OF THE

AMERICAN MATHEMATICAL SOCIETY

Volume 126, Number 3, March 1998, Pages 769-777

S 0002-9939(98)04120-3

\title{
A GENERALIZATION \\ OF LYAPUNOV'S CONVEXITY THEOREM WITH APPLICATIONS IN OPTIMAL STOPPING
}

\author{
ZUZANA KÜHN AND UWE RÖSLER
}

(Communicated by Stanley Sawyer)

\begin{abstract}
Lyapunov proved that the range of $n$ finite measures defined on the same $\sigma$-algebra is compact, and if each measure $\mu_{i}$ also is atomless, then the range is convex. Although both conclusions may fail for measures on different $\sigma$-algebras of the same set, they do hold if the $\sigma$-algebras are nested, which is exactly the setting of classical optimal stopping theory.
\end{abstract}

\section{INTRODUCTION}

In 1940, Lyapunov proved that the range of $n$ finite measures on the same measurable space $(\Omega, \mathcal{A})$ is compact and if the measures are atomless then the range is also convex. There have been many extensions of Lyapunov's convexity theorem; for example see Dubins and Spanier [3] and Dvoretzky, Wald and Wolfowitz [4]. This paper presents a new generalization of this theorem which is of special interest in the theory of optimal stopping. The same conclusions hold under the weakened assumptions of having adapted $\sigma$-algebras $\mathcal{F}_{1} \subseteq \mathcal{F}_{2} \subseteq \cdots \subseteq \mathcal{F}_{n}$, where $\left\{\left(\Omega, \mathcal{F}_{i}, \mu_{i}\right)\right\}_{i=1}^{n}$ is a finite collection of finite measure spaces on the same underlying set $\Omega$. Convexity of the range for general (not necessarily nested) $\sigma$-algebras $\mathcal{F}_{1}, \ldots, \mathcal{F}_{n}$ may fail (Example 2.3 in $[6]$ ).

Definition 1. Let $\mathcal{F}=\left(\mathcal{F}_{1}, \ldots, \mathcal{F}_{n}\right)$. Then the $n$-tuple $\mathbf{A}=\left(A_{1}, \ldots, A_{n}\right) \subseteq \Omega^{n}$ is called an (ordered) $\mathcal{F}$-partition (of $\Omega$ ) if

(i) $A_{i} \in \mathcal{F}_{i}$ for all $i$,

(ii) $A_{i} \cap A_{j}=\emptyset$ if $i \neq j$, and

(iii) $\bigcup_{i=1}^{n} A_{i}=\Omega$.

Note that if $\mathcal{F}_{1}=\mathcal{F}_{2}=\cdots=\mathcal{F}_{n}$, then $\mathbf{A}$ is simply an ordered $\mathcal{F}_{1}$-measurable partition of $\Omega$; i.e. each $A_{i} \in \mathcal{F}_{1}$ and (ii)-(iii) in Definition 1 hold.

Define the vector measure $\mu$, which maps the set of all $\mathcal{F}$-partitions into $\mathbf{R}^{d}$, where $d=n(n+1) / 2$, as follows:

$$
(\mu(\mathbf{A}))_{i j}=\mu_{i}\left(A_{j}\right), \quad 1 \leq j \leq i \leq n .
$$

Received by the editors February 26, 1996 and, in revised form, September 3, 1996.

1991 Mathematics Subject Classification. Primary 28B05; Secondary 60G40.

Key words and phrases. Vector measure, range, optimal stopping.

(c)1998 American Mathematical Society 
Note that in this definition $\mathbf{R}^{d}$ is identified with the set of all lower triangular $n \times n$ matrices; similarly define the "diagonal" vector measure $\mu_{\delta}$ of $\mu$ by

$$
\left(\mu_{\delta}(\mathbf{A})\right)_{i}=\mu_{i}\left(A_{i}\right), \quad i=1, \ldots, n .
$$

The main purpose of this paper is to prove the following theorem.

Theorem 2. For $i=1, \ldots, n$, let $\left(\Omega, \mathcal{F}_{i}, \mu_{i}\right)$ be finite measure spaces with $\mathcal{F}_{1} \subseteq$ $\cdots \subseteq \mathcal{F}_{n}$. Then the set

$$
\{\mu(\mathbf{A}): \mathbf{A} \text { is an } \mathcal{F} \text {-partition }\}
$$

is compact. Moreover, if $\mu_{i}$ is atomless on $\mathcal{F}_{1}$ for all $i$, then the set in (1) is also convex.

The conclusions of Theorem 2 also hold for decreasing $\sigma$-algebras $\mathcal{F}_{1} \supseteq \cdots \supseteq \mathcal{F}_{n}$, with $\mu_{i}$ atomless on $\mathcal{F}_{n}$ instead of $\mathcal{F}_{1}$, and to avoid measurability problems $\mu(\mathbf{A})$ replaced by $\tilde{\mu}(\mathbf{A})$ which is defined by $(\tilde{\mu}(\mathbf{A}))_{i, j}=\mu_{i}\left(A_{j}\right)$ for $1 \leq i \leq j \leq n$. The proof follows analogously.

Theorem 2 has the following immediate corollary which has many applications in optimal stopping theory.

Corollary 3. For $i=1, \ldots, n$ let $\left(\Omega, \mathcal{F}_{i}, \mu_{i}\right)$ be finite measure spaces with $\mathcal{F}_{1} \subseteq$ $\cdots \subseteq \mathcal{F}_{n}$. Then the set

$$
\left\{\mu_{\delta}(\mathbf{A}): \mathbf{A} \text { is an } \mathcal{F} \text {-partition }\right\}
$$

is compact. Moreover, if $\mu_{i}$ is atomless on $\mathcal{F}_{1}$ for all $i$, then the set in $(2)$ is also convex.

Proof. The linear map which assigns to each matrix its diagonal is continuous.

\section{A PRELIMINARY COMPACTNESS RESUlT}

Let $\left\{\left(\Omega, \mathcal{F}_{i}, \mu_{i}\right)\right\}_{i=1}^{n}$ be as in Theorem 2. Define $\bar{\mu}_{i}=\mu_{i}+\ldots+\mu_{n}$ and let $L^{\infty}(\nu, \mathcal{A})$ be the $\mathcal{A}$-measurable $\nu$-a.e. bounded functions. Define

$$
L_{n}=L^{\infty}\left(\bar{\mu}_{1}, \mathcal{F}_{1}\right) \times \cdots \times L^{\infty}\left(\bar{\mu}_{n}, \mathcal{F}_{n}\right)
$$

and

$$
T_{n}: L_{n} \rightarrow \mathbf{R}^{d}, \quad \text { where } \quad d=n(n+1) / 2
$$

by

$$
\left(T_{n}(g)\right)_{i j}=\int g_{j} d \mu_{i}, \quad 1 \leq j \leq i \leq n, \quad \text { where } \quad g=\left(g_{1}, \ldots, g_{n}\right) .
$$

First observe that since $(X \times Y)^{*}$ is isometric to $X^{*} \times Y^{*}$ (see Exercise 4 in [2] for an even more general result), $L_{n}$ is isometrically isometric to the dual of $L^{1}\left(\bar{\mu}_{1}, \mathcal{F}_{1}\right) \times \cdots \times L^{1}\left(\bar{\mu}_{n}, \mathcal{F}_{n}\right)$. Endow $L_{n}$ with the weak* topology, and note that by Radon-Nikodym, $T_{n}$ is a weak* continuous linear mapping.

Throughout this paper let $B(h)$ and $S(h)$ denote the following sets:

$$
B(h)=B_{n}(h)=\left\{g \in L_{n}: \sum_{i=1}^{n} g_{i} \leq h, g_{i} \geq 0, i=1, \ldots, n\right\}
$$

and

$$
S(h)=S_{n}(h)=\left\{g \in L_{n}: \sum_{i=1}^{n} g_{i}=h, g_{i} \geq 0, i=1, \ldots, n\right\}
$$


Lemma 4. For $n \in \mathbf{N}$ and $h \in L^{\infty}\left(\bar{\mu}_{1}, \mathcal{F}_{1}\right)$ the sets $B(h)$ and $S(h)$ are weak* compact in $L_{n}$. In particular, $T_{n}(B(h))$ and $T_{n}(S(h))$ are compact.

Proof. To see that $B(h)$ is weak* compact, it suffices to show that

$$
B(h) \text { is bounded }
$$

and hence, by Alaoglu's theorem, contained in a weak* compact set and that

$$
B(h) \text { is weak }{ }^{*} \text { closed. }
$$

For (3) it must be shown that there exists a constant $K$ (which does not depend on $g$ ) such that

$$
\|g\| \leq K \quad \forall g \in B(h),
$$

where $\|\cdot\|$ is the usual sup norm on $L_{n}$.

$$
\|g\|=\max _{1 \leq i \leq n}\left\|g_{i}\right\| \leq \sum_{i=1}^{n}\left\|g_{i}\right\| \leq n\|h\|<\infty
$$

For (4) let $g=\left(g_{1}, \ldots, g_{n}\right) \in \operatorname{cl}(B(h))$, so there exists a net $\left\langle g_{\gamma}\right\rangle$ in $B(h)$ such that $g_{\gamma}$ converges weak* to $g$. By definition of $B(h)$, for each $\gamma$ the function $g_{\gamma}=\left(g_{\gamma, 1}, \ldots, g_{\gamma, n}\right)$ for some $\left\{g_{\gamma, i}\right\}$ with $\sum_{i=1}^{n} g_{\gamma, i} \leq h$, and with $g_{\gamma, i} \geq 0$ and $g_{\gamma, i} \rightarrow g_{i}$ weak $^{*}$ in $L^{\infty}\left(\bar{\mu}_{i}, \mathcal{F}_{i}\right)$ for all $i \leq n$.

If $\sum_{i=1}^{n} g_{i}>h$ on a set $A$ of $\bar{\mu}_{1}$-positive measure, then weak* ${ }^{*}$ convergence implies that for all $i$

$$
\int g_{\gamma, i} 1_{A} d \bar{\mu}_{i} \rightarrow \int g_{i} 1_{A} d \bar{\mu}_{i}
$$

where $1_{A} \in L^{1}\left(\bar{\mu}_{1}, \mathcal{F}_{1}\right)$.

Then

$$
\int h 1_{A} d \bar{\mu}_{1} \geq \sum_{i=1}^{n} \int g_{\gamma, i} 1_{A} d \bar{\mu}_{1} \rightarrow \sum_{i=1}^{n} \int g_{i} 1_{A} d \bar{\mu}_{1}>\int h 1_{A} d \bar{\mu}_{1}
$$

which is a contradiction. The same argument shows that $g_{i} \geq 0$ for all $i$.

The compactness of $T_{n}(B(h))$ now follows from the weak* continuity of $T_{n}$. The proof for $S(h)$ is similar.

\section{Compactness for ATOMLess measures}

A measurable set $A \in \mathcal{A}$ is an atom for the measure $\nu$ if $\nu(A)>0$ and if for every $B \in \mathcal{A} \quad \nu(A \cap B)$ is either 0 or $\nu(A)$. Note that $\bar{\mu}_{1}$ is atomless on $\mathcal{F}_{1}$ if and only if all $\mu_{i}, i=1, \ldots, n$, are atomless ([3], Lemma 4.1). Notice also that if $\mu_{i}$ is atomless on $\mathcal{F}_{1}$ then it is also atomless on $\mathcal{F}_{i}$. (The converse is not true in general: if $\Omega=[0,1], \mu=$ Lebesgue measure on $[0,1], \mathcal{F}_{1}=\{\emptyset, \Omega\}$, and $\mathcal{F}_{2}=$ Borel $\sigma$-algebra on $[0,1]$, then $\mathcal{F}_{1} \subseteq \mathcal{F}_{2}$, and $\mu$ is atomless on $\mathcal{F}_{2}$, but not on $\mathcal{F}_{1}$.)

Lemma 5. Let $\mu_{1}, \ldots, \mu_{n}$ be finite measures on $\sigma$-algebras $\mathcal{F}_{1} \subseteq \cdots \subseteq \mathcal{F}_{n}$, respectively. Let $\bar{\mu}_{1}$ be atomless on $\mathcal{F}_{1}$ and let $A_{1}$ be $\mathcal{F}_{1}$-measurable and $\left(A_{2}, \ldots, A_{n}\right)$ be an $\left(\mathcal{F}_{2}, \ldots, \mathcal{F}_{n}\right)$-partition. Then there exists an $\mathcal{F}_{1}$-measurable set $B$ with

$$
\mu_{i}\left(B \cap A_{1} \cap A_{j}\right)=\mu_{i}\left(B^{c} \cap A_{1} \cap A_{j}\right), \quad 1 \leq j \leq i \leq n .
$$


Proof. Without loss of generality $\mu_{i}\left(A_{1} \cap A_{j}\right)>0$ for all $i$ and $j \leq i$. Define a map $\phi: \mathcal{F}_{1} \rightarrow \mathbf{R}^{d}$ by

$$
(\phi(B))_{i j}=a_{i j} \mu_{i}\left(B \cap A_{1} \cap A_{j}\right)
$$

with $a_{i j}=1 / \mu_{i}\left(A_{1} \cap A_{j}\right)$. Observe that the map defined on $\mathcal{F}_{j}$ by

$$
C \mapsto a_{i j} \mu_{i}\left(C \cap A_{1} \cap A_{j}\right)
$$

is a probability measure. Since each of these probability measures is atomless on $\mathcal{F}_{1}$, the range of $\phi$ is convex by Lyapunov's theorem. This implies the existence of a set $B_{0} \in \mathcal{F}_{1}$ with $\left(\phi\left(B_{0}\right)\right)_{i j}=\frac{1}{2}$ for all $i$ and $j \leq i$, which satisfies (5).

Recall that a point $x$ in a convex set $D$ is an extreme point of $D$ if there is no representation of the form $x=\lambda y+(1-\lambda) z, \lambda \in(0,1), y \neq z$ and $y, z \in D$.

Proposition 6. Let $\mu_{1}, \ldots, \mu_{n}$ be finite measures on $\sigma$-algebras $\mathcal{F}_{1} \subseteq \ldots \subseteq \mathcal{F}_{n}$, respectively, and let $\bar{\mu}_{1}$ be atomless on $\mathcal{F}_{1}$. Then for $\alpha \in \mathbf{R}^{d}$ and $h \in L^{\infty}\left(\bar{\mu}_{1}, \mathcal{F}_{1}\right)$, the extreme points of

$$
T_{n}^{-1}(\alpha) \cap S(h)
$$

are exactly those functions $g$ in the set $(6)$ for which there is an $\mathcal{F}$-partition $\mathbf{A}$ with

$$
g_{i}=h \cdot 1_{A_{i}}, \quad \bar{\mu}_{i} \text {-a.e., } \quad i=1, \ldots, n .
$$

Proof. By induction on $n$. For $n=1$,

$$
S(h)=\left\{g \in L^{\infty}\left(\bar{\mu}_{1}, \mathcal{F}_{1}\right): 0 \leq g=h\right\},
$$

and the set (6) consists at most of one element, in which case the conclusion is trivial.

Suppose the assertion holds for $n-1$ and let $\alpha \in \mathbf{R}^{d}$. The set (6) is closed in the weak* topology by Lemma 4; assume that it is not empty. Clearly the set (6) is convex because $T_{n}^{-1}(\alpha)$ is an affine hyperplane. Notice that every function $g$ of the form $g_{i}=h \cdot 1_{A_{i}}, \bar{\mu}_{i}$-a.e., where $\left(A_{1}, \ldots, A_{n}\right)$ is an $\mathcal{F}$-partition, is an extreme point in the set (6). Now suppose that $g$ is an extreme point in (6). It remains to show that $g$ has the required form. In particular, $\left(g_{2}, \ldots, g_{n}\right)$ is extremal in the set

$$
\begin{aligned}
M= & \left\{\left(f_{2}, \ldots, f_{n}\right) \in L^{\infty}\left(\bar{\mu}_{2}, \mathcal{F}_{2}\right) \times \cdots \times L^{\infty}\left(\bar{\mu}_{n}, \mathcal{F}_{n}\right):\right. \\
& \left.\sum_{i=2}^{n} f_{i}=h-g_{1}, f_{i} \geq 0 \quad \text { and } \quad \int f_{j} d \mu_{i}=\int g_{j} d \mu_{i} \text { for } 2 \leq j \leq i \leq n\right\} .
\end{aligned}
$$

Note that $h-g_{1} \in L^{\infty}\left(\bar{\mu}_{1}, \mathcal{F}\right)$ and that $\bar{\mu}_{2}$ is atomless on $\mathcal{F}_{2}$. Therefore, by the induction hypothesis there exists an $\left(\mathcal{F}_{2}, \ldots, \mathcal{F}_{n}\right)$-partition $\left(A_{2}, \ldots, A_{n}\right)$ such that

$$
g_{i}=\left(h-g_{1}\right) 1_{A_{i}} \quad \text { for } i=2, \ldots, n .
$$

Define $\mathcal{F}_{1}$-measurable set $D=\left\{\omega \in \Omega: 0<g_{1}(\omega)<h(\omega)\right\}$. If $\bar{\mu}_{1}(D)=0$, then $g_{1}=h 1_{D^{c}} \quad \bar{\mu}_{1}$-a.e., and $g$ has the required representation.

Now suppose, by way of contradiction, that $\bar{\mu}_{1}(D)>0$.

Define a set $D_{\epsilon}:=\left\{\omega \in \Omega: 0<\epsilon<g_{1}(\omega)<h(\omega)-\epsilon\right\}$ for some $\epsilon>0$ such that $\bar{\mu}_{1}\left(D_{\epsilon}\right)>0$. Since $\left(A_{2}, \ldots, A_{n}\right)$ is an $\left(\mathcal{F}_{2}, \ldots, \mathcal{F}_{n}\right)$-partition,

$$
D_{\epsilon}=\bigcup_{i=2}^{n} A_{i} \cap D_{\epsilon} .
$$


Denote $A_{1}=D_{\epsilon}$ and apply Lemma 5 to $A_{1} \in \mathcal{F}_{1}$ and the partition $\left(A_{2}, \ldots, A_{n}\right)$. Choose an $\mathcal{F}_{1}$-measurable set $B$ with

$$
\mu_{i}\left(B \cap A_{1} \cap A_{j}\right)=\mu_{i}\left(B^{c} \cap A_{1} \cap A_{j}\right) \quad \text { for } \quad 1 \leq j \leq i \leq n .
$$

Define $g^{+}=\left(g_{1}^{+}, \ldots, g_{n}^{+}\right)$and $g^{-}=\left(g_{1}^{-}, \ldots, g_{n}^{-}\right)$by

$$
\begin{aligned}
& g_{1}^{+}=g_{1}+\epsilon 1_{B \cap A_{1}}-\epsilon 1_{B^{c} \cap A_{1}}, \\
& g_{1}^{-}=g_{1}-\epsilon 1_{B \cap A_{1}}+\epsilon 1_{B^{c} \cap A_{1}}, \\
& g_{i}^{+}=g_{i}+\epsilon 1_{B^{c} \cap A_{i} \cap A_{1}}-\epsilon 1_{B \cap A_{i} \cap A_{1}}, \\
& g_{i}^{-}=g_{i}-\epsilon 1_{B^{c} \cap A_{i} \cap A_{1}}+\epsilon 1_{B \cap A_{i} \cap A_{1}},
\end{aligned}
$$

for $i=2, \ldots, n$.

By (7), $g^{+}, g^{-} \in T_{n}^{-1}(\alpha)$. By the definition of $D_{\epsilon}$ and

$$
\sum_{i=1}^{n} g_{i}^{+}=\sum_{i=1}^{n} g_{i}^{-}=\sum_{i=1}^{n} g_{i}=h
$$

it follows that $g^{+}, g^{-} \in S(h)$, so $g^{+}, g^{-}$are in the set (6). But this contradicts the extremality of $g$, since $g=\left(g^{+}+g^{-}\right) / 2$ and $g^{+} \neq g^{-}$.

\section{Compactness for general measures}

Theorem 7. Let $\mu_{1}, \ldots, \mu_{n}$ be finite measures on the $\sigma$-algebras $\mathcal{F}_{1} \subseteq \cdots \subseteq \mathcal{F}_{n}$. Then $T_{n}(N)$ is compact, where

$$
N=\left\{g \in L_{n}: g_{i}=1_{A_{i}} \bar{\mu}_{i} \text {-a.e. for some } \mathcal{F} \text {-partition } \mathbf{A}\right\} .
$$

Proof. Let $\left\{B_{k}\right\}_{k=1}^{m}$ be a sequence of disjoint atoms from $\mathcal{F}_{1}$ with respect to $\bar{\mu}_{1}$, where $m \leq \infty$. Set $B=\bigcup_{k=1}^{m} B_{k}$ and assume without loss of generality that $\bar{\mu}_{1}$ is atomless on $B^{c}$. Let $N \mid A$ denote the set $\left\{g 1_{A}: g \in N\right\}$, and note that

$$
g=g 1_{B^{c}}+\sum_{k=1}^{m} g 1_{B_{k}} .
$$

Then

$$
N=N\left|B^{c}+\sum_{k=1}^{m} N\right| B_{k}
$$

and it will be shown

$$
T_{n}(N)=T_{n}\left(N \mid B^{c}\right)+\sum_{k=1}^{m} T_{n}\left(N \mid B_{k}\right) .
$$

If $m<\infty$, then the equality is trivial since $T_{n}$ is linear. Suppose that $m=\infty$ and recall that $T_{n}$ is weak* continuous. Then

$$
\begin{aligned}
T_{n}(N) & =T_{n}\left(N\left|B^{c}+\sum_{k=1}^{\infty} N\right| B_{k}\right)=T_{n}\left(N\left|B^{c}+\lim _{m \rightarrow \infty} \sum_{k=1}^{m} N\right| B_{k}\right) \\
& =\lim _{m \rightarrow \infty} T_{n}\left(N\left|B^{c}+\sum_{k=1}^{m} N\right| B_{k}\right)=\lim _{m \rightarrow \infty}\left(T_{n}\left(N \mid B^{c}\right)+T_{n}\left(\sum_{k=1}^{m} N \mid B_{k}\right)\right) \\
& =T_{n}\left(N \mid B^{c}\right)+\sum_{k=1}^{\infty} T_{n}\left(N \mid B_{k}\right) .
\end{aligned}
$$


First it will be shown that the set $T_{n}\left(N \mid B^{c}\right)$ is compact. Suppose without loss of generality that $B^{c}=\Omega$. It is enough to show that $T_{n}\left(N \mid B^{c}\right)=T_{n}(S(1))$ since the latter set is compact by Lemma 4. Since $N \mid B^{c} \subseteq S(1)$, the inclusion $T_{n}\left(N \mid B^{c}\right) \subseteq$ $T_{n}(S(1))$ is trivial. To obtain the opposite inclusion, pick a point $\alpha \in \mathbf{R}^{d}$ such that $\alpha$ is in the set $T_{n}(S(1))$. It remains to show that $S(1)$ contains some $I=$ $\left(1_{A_{1}}, \ldots, 1_{A_{n}}\right)$ where $\mathbf{A}$ is an $\mathcal{F}$-partition, for then $\alpha_{i j}=\int 1_{A_{j}} d \mu_{i}$ and thus $\alpha$ lies in the set $T_{n}\left(N \mid B^{c}\right)$. The set (6) in Proposition 6 is weak* closed and non-empty. By the Krein-Milman theorem this set is the closure of convex combination of the extremal elements, so in particular, there exists an extremal element, which by Proposition 6 , has the required representation.

It will next be shown that each set $T_{n}\left(N \mid B_{k}\right)$ is also compact.

Denote

$$
T_{n}(g)=\left(R_{n}(g), Q_{n}(g)\right)
$$

where $R_{n}(g)$ denotes the first column of $T_{n}(g)$ and $Q_{n}(g)$ stands for the last $n-1$ columns, so $Q_{n}$ involves only $\mu_{2}, \ldots, \mu_{n}$, and $R_{n}$ deals only with the first coordinate $g_{1}$ of $g$. Since $B_{k}$ is an $\mathcal{F}_{1}$ atom,

$$
R_{n}\left(N \mid B_{k}\right)=\left\{a \in \mathbf{R}^{n}: a_{i}=0 \text { or } a_{i}=\int 1_{B_{k}} d \mu_{i}, \forall i\right\},
$$

so $R_{n}$ can take only finitely many values, i.e.

$$
R_{n}\left(N \mid B_{k}\right)=\{a(1), \ldots, a(l)\} .
$$

Without loss of generality, assume that $a(1), \ldots, a(l)$ are distinct vectors, and define for all $j=1, \ldots, l$

$$
D_{j}=\left\{g \in N \mid B_{k}: R_{n}(g)=a(j)\right\}=R_{n}^{-1}(a(j)) .
$$

Then $N \mid B_{k}=\bigcup_{j=1}^{l} D_{j}$ and

$$
T_{n}\left(N \mid B_{k}\right)=\bigcup_{j=1}^{l} T_{n}\left(D_{j}\right)=\bigcup_{j=1}^{l}\left(a(j), Q_{n}\left(D_{j}\right)\right) .
$$

Since $D_{j}$ is a pre-image of a closed set under the weak* continuous map $R_{n}$, it is weak* closed. Also note that $D_{j}$ is contained in $S(1)$, which by Lemma 4 is weak* compact. Therefore, $D_{j}$ is weak ${ }^{*}$ compact, and since $Q_{n}$ is weak* continuous, $Q_{n}\left(D_{j}\right)$ is compact. This implies that $T_{n}\left(N \mid B_{k}\right)$ is compact.

By Tychonov's theorem (using the continuity of addition and boundedness of $\left.T_{n}(N)\right), T_{n}(N)$ is compact.

The following lemma gives another description of $T_{n}(N)$.

Lemma 8. Under the assumptions of Theorem $7, T_{n}(N)=T_{n}(\bar{N})$, where

$$
\bar{N}=\left\{g \in L_{n}: \sum_{i=1}^{n} g_{i}=1, g_{i} \geq 0, g_{i} \text { has value } 0 \text { or } 1 \text { on atoms of } \mathcal{F}_{i} \bar{\mu}_{i} \text {-a.e. }\right\} .
$$

Proof. The inclusion $T_{n}(N) \subseteq T_{n}(\bar{N})$ is trivial since $N \subseteq \bar{N}$. It remains to show that $T_{n}(\bar{N}) \subseteq T_{n}(N)$. Decompose $\nu=\nu_{a}+\nu_{c}$, where $\nu_{a}$ is purely atomic and $\nu_{c}$ is atomless. The argument for $\nu_{c}$ follows by Proposition 6 applied to $\Omega \backslash\{$ Atoms $\}$.

The argument for $\nu_{a}$ is trivial since $\bar{N} \subseteq N$. 


\section{ProOF OF MAIN THEOREM}

Proof of Theorem 2. By Theorem 7, $T_{n}(N)=\{\mu(\mathbf{A}): \mathbf{A}$ is an $\mathcal{F}$-partition $\}$ is compact.

Further $T_{n}(N)=T_{n}(\bar{N})$ by Lemma 8 . If $\bar{\mu}_{1}$ is atomless, then $\bar{N}$ is convex and so is $T(\bar{N})$.

Note that in general the set $T_{n}(N)$ may not be convex, since if $\mathcal{F}_{1}=\mathcal{F}_{2}=\{\emptyset, \Omega\}$ and $\mu_{1}=\mu_{2}$ are probability measures on $\mathcal{F}_{1}$, then $T_{2}(N)=\{(1,1,0),(0,0,1)\}$.

The next example shows that it is not enough to consider only $\mathcal{F}_{1}$-measurable partitions $\left(A_{1}, \ldots, A_{n}\right)$ since the range in this case is different from the set (2).

Example 9. Let $\Omega=[0,1] \times[0,1]$. Denote by $\mathcal{B}[0,1]$ the Borel $\sigma$-algebra on $[0,1]$ and let $\mathcal{F}_{1}=\mathcal{B}[0,1] \times\{\emptyset,[0,1]\}$. Let $\mathcal{F}_{2}=\mathcal{F}_{3}$ be the usual Borel $\sigma$-algebras on $\Omega$. Denote by $\lambda$ Lebesgue measure on $\mathcal{B}([0,1])$ and by $\delta_{[0,1]}$ Dirichlet measure on $\{\emptyset,[0,1]\}$. Define $\mu_{1}, \mu_{2}$ and $\mu_{3}$ for all $B=B_{1} \times B_{2}$ in $\mathcal{F}_{1}, \mathcal{F}_{2}, \mathcal{F}_{3}$ respectively by

$$
\begin{aligned}
& \mu_{1}(B)=\lambda\left(B_{1}\right) \times \delta_{[0,1]}\left(B_{2}\right) \quad \text { for all } \quad B \in \mathcal{F}_{1}, \\
& \mu_{2}(B)=2 \lambda\left(B_{1}\right) \times \lambda\left(\left[0, \frac{1}{2}\right) \cap B_{2}\right) \quad \text { for all } \quad B \in \mathcal{F}_{2}, \\
& \mu_{3}(B)=2 \lambda\left(B_{1}\right) \times \lambda\left(\left[\frac{1}{2}, 1\right) \cap B_{2}\right) \quad \text { for all } \quad B \in \mathcal{F}_{3} .
\end{aligned}
$$

Then

$(0,1,1) \notin\left\{\mu_{\delta}(\mathbf{A}): \mathbf{A}\right.$ is an ordered partition of $\Omega$ such that $\left.A_{i} \in \mathcal{F}_{1}\right\}$,

and for $\mathbf{A}=\left(\emptyset \times \emptyset,[0,1] \times\left[0, \frac{1}{2}\right),[0,1] \times\left[\frac{1}{2}, 1\right]\right)$,

$$
(0,1,1) \in\left\{\mu_{\delta}(\mathbf{A}): \mathbf{A} \text { is an } \mathcal{F} \text {-partition }\right\} .
$$

\section{Applications}

Throughout this section $X_{1}, \ldots, X_{n}$ is a sequence of random variables defined on the same probability space $(\Omega, \mathcal{F}, P)$. Recall that a stopping time $t$ for $X_{1}, \ldots, X_{n}$ is a random variable with values in $\{1, \ldots, n\}$ such that $\{t=i\} \in \mathcal{F}_{i}$ for all $i \leq n$, where $\mathcal{F}_{i}=\sigma\left(X_{1}, \ldots, X_{i}\right)$. Let

$$
\mu_{i}(F)=\int_{F} X_{i}
$$

for all $i \leq n$ and all $F \in \mathcal{F}_{i}$, and

$$
R(\vec{X})=\left\{\left(\int_{t=1} X_{1}, \ldots, \int_{t=n} X_{n}\right): t \text { is a stopping time for } X_{1}, \ldots, X_{n}\right\} .
$$

Note that the sets $(2)$ and $R(\vec{X})$ are identical. To see that $R(\vec{X})$ is contained in the set (2), let $t$ be an arbitrary stopping time. Denote $A_{i}=\{t=i\}$, for all $i=1, \ldots, n$, and notice that $A_{i} \in \mathcal{F}_{i}, A_{i} \cap A_{j}=\emptyset$ if $i \neq j$ and $\cup_{i=1}^{n} A_{i}=\Omega$. The opposite inclusion follows also easily. Let $\mathbf{A}$ be an $\mathcal{F}$-partition such that $\left(\mu_{1}\left(A_{1}\right), \ldots, \mu_{n}\left(A_{n}\right)\right)$ is in the set (2), and define the stopping time $t$ by $\{t=i\}=$ $A_{i}$.

This gives an equivalent formulation of Corollary 3 in the language of optimal stopping of random variables.

Corollary $3^{\prime}$. If $X_{1}, \ldots, X_{n}$ are integrable, then the set $R(\vec{X})$ is a compact subset of $\mathbf{R}^{n}$. 
A well known fact from optimal stopping theory (cf. [1]) which is proven by backward induction is that if $X_{1}, \ldots, X_{n}$ are integrable, then

$$
\sup \left\{E X_{t}: t \text { is a stopping time for } X_{1}, \ldots, X_{n}\right\}
$$

is attained. This also follows immediately from Corollary $3^{\prime}$, which yields much more general result.

Corollary 10. Let $\phi: \mathbf{R}^{n} \rightarrow \mathbf{R}$ be a continuous function and $X_{1}, \ldots, X_{n}$ be integrable random variables on $(\Omega, \mathcal{F}, P)$. Then

$$
\left\{\phi\left(\int_{t=1} X_{1}, \ldots, \int_{t=n} X_{n}\right): t \text { is a stopping time for } X_{1}, \ldots, X_{n}\right\}
$$

is a compact subset of $\mathbf{R}$.

Setting $\phi_{1}\left(x_{1}, \ldots, x_{n}\right)=\sum_{i=1}^{n} x_{i}$ in Corollary 10 gives the existence of an optimal stopping rule $t$ which maximizes $E X_{t}$, since $E X_{t}=\int_{t=1} X_{1}+\ldots+\int_{t=n} X_{n}$ and since addition is a continuous function. Other examples of typical objective functions $\phi$ satisfying these hypothesis are $\phi(\vec{x})=\min _{1 \leq i \leq n} x_{i}, \quad \phi(\vec{x})=\overline{\operatorname{med}}_{1 \leq i \leq n} x_{i}$, or $\phi(\vec{x})=\prod_{i=1}^{n} x_{i}$. (See [6] for more details.)

Corollary $3^{\prime}$ may be applied to the ranges of randomized stopping times. Define a random stopping time as a random variable with values in $\{1, \ldots, n\}$ such that $\{t=i\} \in \sigma\left(X_{1}, U_{1}, \ldots, X_{n}, U_{n}\right)$, where $U_{1}, \ldots, U_{n}$ are i.i.d. $U[0,1]$ random variables that are independent of $X_{1}, \ldots, X_{n}$. Let $\mathcal{T}_{t}$ denote the set of all randomized stopping times and

$$
G_{n}(\vec{X})=\left\{\left(\int_{t=1} X_{1}, \ldots, \int_{t=n} X_{n}\right): t \in \mathcal{T}_{t}\right\}
$$

denote the randomized stopping time range. Letting $\mathcal{F}_{i}=\sigma\left(X_{1}, Y_{1}, \ldots, X_{i}, Y_{i}\right)$ and $\mu_{i}(F)=\int_{F} X_{i}$ for all $i$, Corollary $3^{\prime}$ implies the compactness of $G_{n}(\vec{X})$, proving a conjecture of Gouweleeuw [5].

\section{ACKNOWLEDGMENTS}

The authors would like to thank Professor T.P. Hill, W. Kuhn, and an anonymous referee for suggestions which improved the presentation of this paper.

\section{REFERENCES}

1. Chow, Y. S., Robbins, H. and Siegmund, D., Great Expectations: The Theory of Optimal Stopping, Houghton Mifflin, Boston, 1971. MR 48:10007

2. Conway, J. B., A Course in Functional Analysis, Springer-Verlag, New York, 1990, p. 77. MR 91e: 46001

3. Dubins, L. and Spanier, E., How to cut a cake fairly, Amer. Math. Monthly (68) (1961), 1-17. MR 23:B2068

4. Dvoretzky, A., Wald, A. and Wolfowitz, J., Relations among certain ranges of vector measures, Pacific J. Math. (1) (1951), 59-74. MR 13:331f

5. Gouweleeuw, J. M., On ranges of vector measures and optimal stopping, Ph.D. Thesis, Vrije Universiteit Amsterdam, 1994.

6. Hill, T. P., Kennedy, D. P., Optimal stopping problems with generalized objective functions, J. Appl. Prob (27) (1990), 828-838. MR 91j:60079

7. Lyapunov, A., Sur les fonctions-vecteurs completement additives, Bull. Acad. Sci. URSS (6) (1940), 465-478. 
8. Rösler, U., More on how to cut a cake fairly, Technical report, Uppsala University (1990:8) (1990).

9. Rudin, W., Functional Analysis, Tata McGraw-Hill, 1973. MR 51:1315

School of Mathematics, Georgia Institute of Technology, Atlanta, Georgia 30332

E-mail address: gt9843a@prism.gatech.edu

Current address: Brinkmannstr. 4, 12169 Berlin, Bermany

Mathematisches Seminar der CAU Kiel, Christian-Albrechts-Universität zU Kiel, Ludewig-Meyn-Str. 4, 24098 Kiel, Germany

E-mail address: nms34@rz.uni-kiel.d400.de 